\title{
“Percepción de la Temperatura Climatológica en el Turismo y en Residentes de la Isla de Mallorca"
}

\author{
José M. - Torrens Calleja \\ Climatología, hidrología, riesgos naturales y territorio (CLIMARIS) \\ Departamento Geografía UIB
}

\begin{abstract}
RESUMEN
El presente artículo de percepción climatológica se basa en encuestas realizadas a turistas que han pernoctado en la Ciudad de Palma y han explorado la isla de Mallorca. Otros encuestados vinculantes han sido grupos de estudiantes de Geografía de Mallorca. Se interpretan los datos de percepción del turismo y población local. Al igual los datos de la serie climatológica del puerto y aeropuerto de la ciudad de Palma de Mallorca. La metodología se realiza con entrevistas personales dando a conocer su vinculación con el saber del clima que se percibe en Mallorca. Los resultados muestran dicha percepción que se puede ajustar o no a la realidad climatológica. El conocimiento de la isla se interpreta mediante preconceptos de la información meteorológica previa al realizar el viaje, por la experiencia en la isla y también por experiencia de visitas anteriores al territorio de Mallorca.
\end{abstract}

\section{INTRODUCCIÓN}

El concepto principal de investigación es conocer la percepción de la climatología mediante el conocimiento del turismo y de los residentes de la isla. En cuanto al estado del arte de la infestación se pueden citar artículos científicos que acercan a la interpretación del nuestro territorio. La percepción está tratada en forma inclusiva en artículos sobre la percepción climatológica. Existen autores que tratan la percepción de la climatología dentro del territorio español. Los autores son los siguientes; Alomar, G (2010); González, I.C (2009); López, F (1995); Martín Vide, J (1990); Moreno, M.C (1987). Entre la temática reinante de la percepción del clima, se tratan las brisas dentro del territorio insular, se estudia la percepción del clima en las ciudades españolas, más concretamente la precipitación en las ciudades de Ávila y Barcelona, incluso el clima urbano de la ciudad de Zaragoza. Hay que tener en cuenta que la percepción puede ser muy cercana a la percepción de la población residente en Mallorca. La investigación es novedosa e interesante en su modo de innovación sobre la percepción del clima. Tiene múltiples facetas para desarrollar y extrapolar la investigación a diferentes ciudades turísticas. El sector económico de mayor importancia en las Islas Baleares es el turismo nacional y extranjero. Al igual, la climatología es un factor determinante para las actividades turísticas. En el turismo isleño vacacional la temperatura favorece el desarrollo de frecuentación de las playas de la costa, las actividades náuticas y las actividades de ocio al aire libre. Dentro de la investigación se realiza el estudio sobre los turistas, los cuales son encuestados en sus lugares de descanso, con diversas preguntas sobre su percepción de las temperaturas que se registran en la isla y además de poder evaluar si perciben de forma coherente el clima de Mallorca. La percepción puede ser positiva para conocer el clima en el territorio de la isla. La percepción es subjetiva según la consideración del propio encuestado, que tanto puede ser acertada como totalmente errónea.

\section{LOCALIZACIÓN}

La localización de la investigación es en el archipiélago Balear (España). Concretamente en la capital Balear, Palma de Mallorca. La población de Palma es de 409.661 residentes, según el 
padrón municipal, IBESTAT (2018). El puerto de Porto Pi, se encuentra en la ribera de la ciudad, dentro del entramado urbano. El aeropuerto de Palma se sitúa a las afueras del entramado urbano de la ciudad. La isla de Mallorca en el transcurso del año 2017, ha recibido el número de visitas de 11.636.313 turistas, según AETIB, (2017). El clima es de características de Csa, según la clasificación de Köpper. Es clima mediterráneo típico, se caracteriza por veranos secos y calurosos, con temperaturas medias por encima de los $22{ }^{\circ} \mathrm{C}$ y con inviernos húmedos y lluviosos, con temperaturas suaves. Un grupo de las entrevistas a turistas se han llevado a cabo en el Hotel Belvedere de Palma y las encuestas a estudiantes en la Universidad de las Islas Baleares, localizada en Palma de Mallorca. Un grupo de las entrevistas a estudiantes de secundaria se han realizado en la Universidad de las Islas Baleares, como dato a observar en el curso académico 2013-14 habían estado matriculados 11.355 alumnos de Bachiller.

\section{METODOLOGÍA}

El método principal de causa en la investigación ha sido llegar al turista con entrevistas personales. La localización del estudio está en el hotel Belvedere de la ciudad de Palma. La muestra de encuestados tiene la diversidad de nacionalidades de la comunidad europea. Siendo dichos encuestados los turistas más asiduos a Mallorca. Las preguntas relevantes han sido sobre la percepción de la temperatura climática en la isla. Las encuestas de percepción se han basado en los siguientes conceptos climatológicos, siendo enunciadas adecuadas para la comprensión y análisis. Dichas preguntas caracterizadas son las siguientes: la percepción del mes más frío y de calor del año, la percepción de la oscilación térmica diaria, la percepción de las temperaturas máximas y mínimas y la percepción de la hora del día en que se registran las temperaturas máximas y mínimas de la jornada. Como ejemplo a expresar dentro del método se destaca que si conocen o perciben la temperatura máxima o mínima que se registra en Mallorca. Una segunda parte de la metodología es analizar la serie climatológica de la estación del puerto y aeropuerto de la ciudad de Palma de Mallorca. En las anteriores series climatológicas mencionadas se muestran los registros históricos de la variable de las temperaturas acontecidos en la ciudad.

\section{RESULTADOS}

Todos los resultados se presentan en una tabla de percepción por parte los encuestados. Del mismo modo se analizan los datos climatológicos de las series. De este modo se puede graduar el ajuste de la percepción de la variable temperatura climatológica de Mallorca. 
Calleja, J. M. T. (2020). "Percepción de la Temperatura Climatológica en el Turismo y en Residentes de la Isla de Mallorca". Advances in Social Sciences Research Journal, 7(1) 236-246.

Tabla 1. Percepción del mes frío del año y los resultados de las series climáticas 1981-2010

\begin{tabular}{cccccccccccccc}
\hline FRIO & $\mathbf{E}$ & $\mathbf{F}$ & $\mathbf{M}$ & $\mathbf{A}$ & $\mathbf{M}$ & $\mathbf{J}$ & $\mathbf{J}$ & $\mathbf{A}$ & $\mathbf{S}$ & $\mathbf{0}$ & $\mathbf{N}$ & $\mathbf{D}$ & Total \\
\hline Turistas n & 182 & 88 & 7 & 4 & 0 & 0 & 3 & 2 & 7 & 5 & 18 & 95 & 411 \\
\hline Porcentaje & 44,3 & 21,4 & 1,7 & 1,0 & 0,0 & 0,0 & 0,7 & 0,5 & 1,7 & 1,2 & 4,4 & 23,1 & 100 \\
\hline Estudiantes n & 178 & 133 & 3 & 1 & 0 & 0 & 0 & 0 & 0 & 0 & 4 & 39 & 358 \\
\hline Porcentaje & 49,7 & 37,2 & 0,8 & 0,3 & 0,0 & 0,0 & 0,0 & 0,0 & 0,0 & 0,0 & 1,1 & 10,9 & 100 \\
\hline Hombres n & 196 & 123 & 4 & 2 & 0 & 0 & 0 & 2 & 3 & 1 & 10 & 48 & 389 \\
\hline Porcentaje & 50,4 & 31,6 & 1,0 & 0,5 & 0,0 & 0,0 & 0,0 & 0,5 & 0,8 & 0,3 & 2,6 & 12,3 & 100 \\
\hline Mujeres n & 251 & 192 & 7 & 1 & 0 & 0 & 3 & 0 & 4 & 4 & 18 & 102 & 582 \\
\hline Porcentaje & 43,1 & 33,0 & 1,2 & 0,2 & 0,0 & 0,0 & 0,5 & 0,0 & 0,7 & 0,7 & 3,1 & 17,5 & 100 \\
\hline Inglaterra n & 63 & 23 & 2 & 0 & 0 & 0 & 1 & 0 & 1 & 4 & 7 & 29 & 130 \\
\hline Porcentaje & 48,5 & 17,7 & 1,5 & 0,0 & 0,0 & 0,0 & 0,8 & 0,0 & 0,8 & 3,1 & 5,4 & 22,3 & 100 \\
\hline Alemania n & 24 & 15 & 1 & 0 & 0 & 0 & 1 & 1 & 5 & 0 & 3 & 14 & 64 \\
\hline Porcentaje & 37,5 & 23,4 & 1,6 & 0,0 & 0,0 & 0,0 & 1,6 & 1,6 & 7,8 & 0,0 & 4,7 & 21,9 & 100 \\
\hline Francia n & 13 & 8 & 1 & 1 & 0 & 0 & 0 & 0 & 0 & 0 & 5 & 20 & 48 \\
\hline Porcentaje & 27,1 & 16,7 & 2,1 & 2,1 & 0,0 & 0,0 & 0,0 & 0,0 & 0,0 & 0,0 & 10,4 & 41,7 & 100 \\
\hline Bélgica n & 14 & 9 & 0 & 0 & 0 & 0 & 0 & 0 & 0 & 0 & 0 & 12 & 35 \\
\hline Porcentaje & 40,0 & 25,7 & 0,0 & 0,0 & 0,0 & 0,0 & 0,0 & 0,0 & 0,0 & 0,0 & 0,0 & 34,3 & 100 \\
\hline España n & 21 & 9 & 0 & 0 & 0 & 0 & 0 & 0 & 0 & 1 & 1 & 7 & 39 \\
\hline Porcentaje & 53,8 & 23,1 & 0,0 & 0,0 & 0,0 & 0,0 & 0,0 & 0,0 & 0,0 & 2,6 & 2,6 & 17,9 & 100 \\
\hline Tínima media Puerto & 8,3 & 8,4 & 9,6 & 11,7 & 15,1 & 18,9 & 21,9 & 22,5 & 19,9 & 16,6 & 12,3 & 9,7 & 14,6 \\
\hline ma media Aeropuerto & 3,8 & 4,0 & 5,2 & 7,4 & 11,3 & 15,4 & 18,3 & 18,9 & 16,5 & 13,1 & 8,3 & 5,4 & 10,6 \\
\hline
\end{tabular}

Los turistas perciben el mes de enero como el más frío del año, seguido del mes de diciembre. Los hombres, los estudiantes y el Reino Unido, presentan el grado superior de la percepción correcta. Francia percibe el mes de diciembre como el más frío, seguido del mes de enero. España, los estudiantes, los hombres, las mujeres y Alemania perciben el mes de enero primero, seguido de febrero. Los resultados de la serie climática de las dos localizaciones, muestran que en el Puerto de Palma el mes frío es enero, con escasa diferencia de febrero por $0,1^{\circ} \mathrm{C}$. En el aeropuerto de Palma, los resultados son semejantes, enero se diferencia de febrero en $0,2^{\circ} \mathrm{C}$. 
Tabla 2. Percepción del mes de calor y los resultados de las series climáticas 1981-2010

\begin{tabular}{|c|c|c|c|c|c|c|c|c|c|c|c|c|c|}
\hline CALUROSO & $\mathbf{E}$ & $\mathbf{F}$ & $\mathbf{M}$ & A & $\mathbf{M}$ & J & J & A & $\mathbf{S}$ & $\mathbf{0}$ & $\mathbf{N}$ & D & Total \\
\hline Turistas $\mathrm{n}$ & 0 & 1 & 0 & 2 & 4 & 17 & 176 & 205 & 6 & 0 & 0 & 0 & 411 \\
\hline Porcentaje & 0,0 & 0,2 & 0,0 & 0,5 & 1,0 & 4,1 & 42,8 & 49,9 & 1,5 & 0,0 & 0,0 & 0,0 & 100 \\
\hline Estudiantes n & 0 & 0 & 0 & 0 & 0 & 5 & 122 & 236 & 0 & 0 & 0 & 0 & 363 \\
\hline Porcentaje & 0,0 & 0,0 & 0,0 & 0,0 & 0,0 & 1,4 & 33,6 & 65,0 & 0,0 & 0,0 & 0,0 & 0,0 & 100 \\
\hline Hombres n & 2 & 1 & 2 & 5 & 3 & 11 & 163 & 190 & 3 & 2 & 4 & 3 & 389 \\
\hline Porcentaje & 0,5 & 0,3 & 0,5 & 1,3 & 0,8 & 2,8 & 41,9 & 48,8 & 0,8 & 0,5 & 1,0 & 0,8 & 100 \\
\hline Mujeres n & 4 & 6 & 5 & 3 & 6 & 16 & 193 & 334 & 10 & 5 & 3 & 4 & 589 \\
\hline Porcentaje & 0,7 & 1,0 & 0,8 & 0,5 & 1,0 & 2,7 & 32,8 & 56,7 & 1,7 & 0,8 & 0,5 & 0,7 & 100 \\
\hline Inglaterra $\mathrm{n}$ & 0 & 0 & 0 & 1 & 1 & 6 & 42 & 81 & 1 & 0 & 0 & 0 & 132 \\
\hline Porcentaje & 0,0 & 0,0 & 0,0 & 0,8 & 0,8 & 4,5 & 31,8 & 61,4 & 0,8 & 0,0 & 0,0 & 0,0 & 100 \\
\hline Alemania $\mathrm{n}$ & 0 & 1 & 0 & 0 & 0 & 1 & 29 & 30 & 1 & 0 & 0 & 0 & 62 \\
\hline Porcentaje & 0,0 & 1,6 & 0,0 & 0,0 & 0,0 & 1,6 & 46,8 & 48,4 & 1,6 & 0,0 & 0,0 & 0,0 & 100 \\
\hline Francia n & 0 & 0 & 0 & 0 & 0 & 1 & 18 & 23 & 1 & 0 & 0 & 0 & 43 \\
\hline Porcentaje & 0,0 & 0,0 & 0,0 & 0,0 & 0,0 & 2,3 & 41,9 & 53,5 & 2,3 & 0,0 & 0,0 & 0,0 & 100 \\
\hline Bélgica n & 0 & 0 & 0 & 0 & 0 & 1 & 19 & 15 & 0 & 0 & 0 & 0 & 35 \\
\hline Porcentaje & 0,0 & 0,0 & 0,0 & 0,0 & 0,0 & 2,9 & 54,3 & 42,9 & 0,0 & 0,0 & 0,0 & 0,0 & 100 \\
\hline España n & 0 & 0 & 0 & 0 & 0 & 2 & 19 & 17 & 1 & 0 & 0 & 0 & 39 \\
\hline Porcentaje & 0,0 & 0,0 & 0,0 & 0,0 & 0,0 & 5,1 & 48,7 & 43,6 & 2,6 & 0,0 & 0,0 & 0,0 & 100 \\
\hline T máxima media Puerto & 15,4 & 15,5 & 17,2 & 19,2 & 22,5 & 26,5 & 29,4 & 29,8 & 27,1 & 23,7 & 19,3 & 16,5 & 21,8 \\
\hline T máxima media Aeropuerto & 15,2 & 15,4 & 17,5 & 19,8 & 23,7 & 28,1 & 31,2 & 31,3 & 27,9 & 23,9 & 19,0 & 16,1 & 22,4 \\
\hline
\end{tabular}

Los turistas perciben respecto al mes con mayores temperaturas, los meses de agosto y de julio, como el primer y segundo mes calurosos. La impresión de los turistas ingleses, de los alemanes y de los franceses es el mes de agosto, y consecutivamente es en mes de julio. Los estudiantes perciben el mes de agosto como el más caluroso, seguido de julio. Los turistas belgas y españoles tienen la percepción de julio, y después que agosto presenta las altas temperaturas del año. La percepción entre hombres y mujeres presenta similitud, los meses de agosto y julio, respectivamente por grado de percepción. Los resultados de las series climáticas presentan el mes de agosto como el más caluroso, a escasa diferencia del mes de julio, en décimas de grados. La diferencia entre las temperaturas de los dos meses es de mayor en el puerto, respecto al aeropuerto. 
Calleja, J. M. T. (2020). "Percepción de la Temperatura Climatológica en el Turismo y en Residentes de la Isla de Mallorca". Advances in Social Sciences Research Journal, 7(1) 236-246.

Tabla 3. Percepción de la oscilación térmica diaria y los resultados de las series climáticas 1981-2010

\begin{tabular}{|c|c|c|c|c|c|c|c|c|c|c|c|c|c|}
\hline OSCILACIÓN TÉRMICA & $\mathbf{E}$ & $\mathbf{F}$ & $\mathbf{M}$ & $\mathbf{A}$ & $\mathbf{M}$ & J & J & $\mathbf{A}$ & $S$ & $\mathbf{0}$ & $\mathbf{N}$ & D & Total \\
\hline Turistas $\mathrm{n}$ & 8 & 12 & 21 & 53 & 47 & 28 & 14 & 29 & 38 & 29 & 12 & 9 & 300 \\
\hline Porcentaje & 2,7 & 4,0 & 7,0 & 17,7 & 15,7 & 9,3 & 4,7 & 9,7 & 12,7 & 9,7 & 4,0 & 3,0 & 100 \\
\hline Estudiantes n & 18 & 26 & 71 & 44 & 38 & 16 & 15 & 10 & 39 & 22 & 19 & 16 & 334 \\
\hline Porcentaje & 5,4 & 7,8 & 21,3 & 13,2 & 11,4 & 4,8 & 4,5 & 3,0 & 11,7 & 6,6 & 5,7 & 4,8 & 100 \\
\hline Hombres $\mathrm{n}$ & 20 & 18 & 55 & 56 & 45 & 17 & 13 & 16 & 44 & 22 & 14 & 15 & 335 \\
\hline Porcentaje & 6,0 & 5,4 & 16,4 & 16,7 & 13,4 & 5,1 & 3,9 & 4,8 & 13,1 & 6,6 & 4,2 & 4,5 & 100 \\
\hline Mujeres n & 19 & 31 & 82 & 74 & 58 & 33 & 22 & 29 & 51 & 47 & 29 & 13 & 488 \\
\hline Porcentaje & 3,9 & 6,4 & 16,8 & 15,2 & 11,9 & 6,8 & 4,5 & 5,9 & 10,5 & 9,6 & 5,9 & 2,7 & 100 \\
\hline Inglaterra $\mathrm{n}$ & 4 & 10 & 3 & 14 & 25 & 12 & 7 & 16 & 21 & 7 & 3 & 3 & 125 \\
\hline Porcentaje & 3,2 & 8 & 2,4 & 11,2 & 20 & 9,6 & 5,6 & 12,8 & 16,8 & 5,6 & 2,4 & 2,4 & 100 \\
\hline Alemania $\mathrm{n}$ & 0 & 0 & 5 & 10 & 5 & 2 & 2 & 0 & 2 & 1 & 0 & 0 & 27 \\
\hline Porcentaje & 0,0 & 0,0 & 18,5 & 37,0 & 18,5 & 7,4 & 7,4 & 0,0 & 7,4 & 3,7 & 0,0 & 0,0 & 100 \\
\hline Francia n & 2 & 1 & 0 & 1 & 0 & 0 & 1 & 0 & 0 & 0 & 0 & 0 & 5 \\
\hline Porcentaje & 40,0 & 20,0 & 0,0 & 20,0 & 0,0 & 0,0 & 20,0 & 0,0 & 0,0 & 0,0 & 0,0 & 0,0 & 100 \\
\hline Bélgica $\mathrm{n}$ & 0 & 0 & 2 & 6 & 2 & 2 & 1 & 0 & 6 & 4 & 1 & 1 & 25 \\
\hline Porcentaje & 0,0 & 0,0 & 8,0 & 24,0 & 8,0 & 8,0 & 4,0 & 0,0 & 24,0 & 16,0 & 4,0 & 4,0 & 100 \\
\hline España n & 0 & 0 & 2 & 6 & 5 & 5 & 2 & 4 & 3 & 5 & 2 & 1 & 35 \\
\hline Porcentaje & 0,0 & 0,0 & 5,7 & 17,1 & 14,3 & 14,3 & 5,7 & 11,4 & 8,6 & 14,3 & 5,7 & 2,9 & 100 \\
\hline $\begin{array}{l}\text { Oscilación T media max-min } \\
\text { Puerto }\end{array}$ & 7,1 & 7,1 & 7,6 & 7,5 & 7,4 & 7,6 & 7,5 & 7,3 & 7,2 & 7,1 & 7 & 6,8 & 7,2 \\
\hline $\begin{array}{l}\text { Oscilación T media max-min } \\
\text { Aeropuerto }\end{array}$ & 11,4 & 11,4 & 12,3 & 12,4 & 12,4 & 12,7 & 12,9 & 12,4 & 11,4 & 10,8 & 10,7 & 10,7 & 11,8 \\
\hline
\end{tabular}

La percepción de los turistas en general es que la oscilación térmica es acusada en el mes de abril, y también en el mes de mayo. La percepción por procedencia de países es diferente. En Reino Unido tienen la percepción del mes de septiembre y el mes de agosto. En Alemania las valoraciones son el mes de abril, seguido por los meses de marzo y mayo. Los turistas de Francia perciben el mes enero. En Bélgica son los meses de abril y de septiembre. En España se capta el mes de abril, seguido equitativamente por los meses de mayo, junio y octubre. La percepción de los estudiantes son los meses de marzo y de abril, por orden respectivamente. Los hombres perciben el mes primero de abril, seguido de marzo. Las mujeres perciben, en primer lugar marzo y en segundo lugar abril. En los resultados de las series climáticas de la oscilación térmica entre las temperaturas máximas y mínimas, se destaca los meses con elevado contraste térmico. En el puerto destacan los meses de mayo y de junio, con la misma variación de temperatura. En el aeropuerto destaca el mes de julio, seguido por el mes de junio, con contrastes térmicos similares. 
Tabla 4. Percepción de las temperaturas máximas y los resultados de las series climáticas 1954-2015

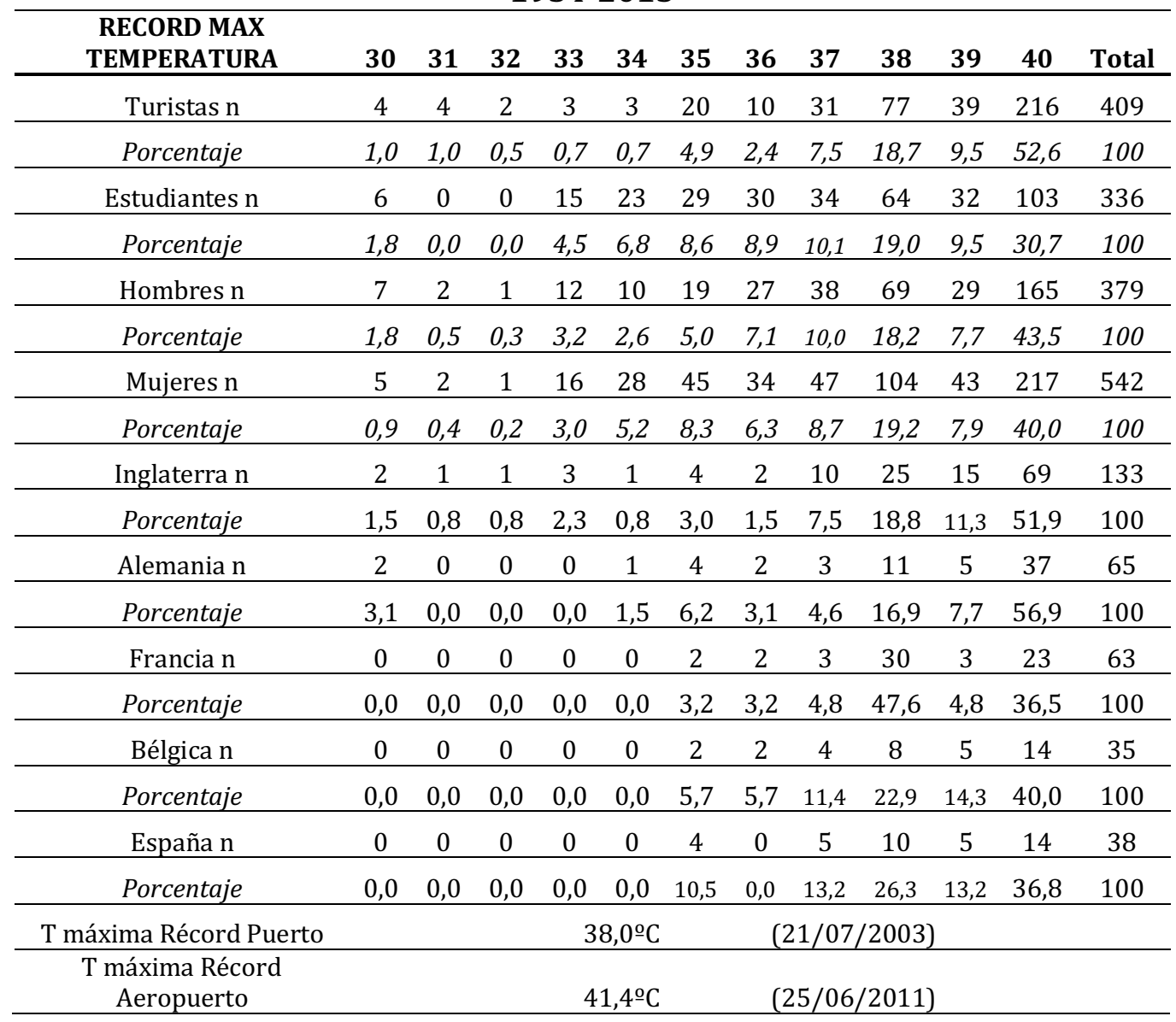

La percepción del registro de temperatura máxima en los turistas en general es en la mayoría de $40^{\circ} \mathrm{C}$, seguido de la percepción de la temperatura de $38^{\circ} \mathrm{C}$. En la impresión de las temperaturas por la procedencia de país, coinciden con la idea general, a excepción de Francia, que perciben el récord de temperatura en $38^{\circ} \mathrm{C}$, seguido del registro de $40^{\circ} \mathrm{C}$. Los estudiantes perciben en más porcentaje $40^{\circ} \mathrm{C}$, seguido del segundo porcentaje de $38^{\circ} \mathrm{C}$. La percepción de los hombres y mujeres, es similar a la tendencia de la percepción general, la primera percepción es de $40^{\circ} \mathrm{C}$, seguida la segunda percepción de $38^{\circ} \mathrm{C}$. Los resultados de las series climáticas en cuanto a los récords de temperatura máxima de las dos estaciones meteorológicas representadas son los siguientes: en el puerto de Palma se ha registrado la temperatura de $38^{\circ} \mathrm{C}$, en el aeropuerto de Palma se ha registrado la temperatura de $41,4^{\circ} \mathrm{C}$. La diferencia de los resultados entre las dos localizaciones, marcan una diferencia en los microclimas, entre estaciones situadas en diversas características territoriales. Zona urbana marítima y zona rural marítima. 
Calleja, J. M. T. (2020). "Percepción de la Temperatura Climatológica en el Turismo y en Residentes de la Isla de Mallorca". Advances in Social Sciences Research Journal, 7(1) 236-246.

Tabla 5. Percepción de las temperaturas mínimas y los resultados de las series climáticas 1954-2015

\begin{tabular}{|c|c|c|c|c|c|c|c|c|c|c|c|c|}
\hline $\begin{array}{c}\text { RECORD MIN } \\
\text { TEMPERATURA } \\
\end{array}$ & -5 & -4 & -3 & -2 & -1 & $\mathbf{0}$ & 1 & 2 & 3 & 4 & 5 & Total \\
\hline Turistas n & 32 & 20 & 40 & 57 & 36 & 36 & 17 & 34 & 32 & 24 & 75 & 403 \\
\hline Porcentaje & 7,9 & 5,0 & 9,9 & 14,1 & 8,9 & 8,9 & 4,2 & 8,4 & 7,9 & 5,9 & 18,6 & 100 \\
\hline Estudiantes n & 64 & 38 & 59 & 53 & 33 & 34 & 10 & 18 & 14 & 8 & 15 & 346 \\
\hline Porcentaje & 18,5 & 11,0 & 17,1 & 15,3 & 9,5 & 9,8 & 2,9 & 5,2 & 4,0 & 2,3 & 4,3 & 100 \\
\hline Hombres $\mathrm{n}$ & 63 & 26 & 43 & 46 & 38 & 37 & 15 & 30 & 22 & 15 & 46 & 381 \\
\hline Porcentaje & 16,5 & 6,8 & 11,3 & 12,1 & 10,0 & 9,7 & 3,9 & 7,9 & 5,8 & 3,9 & 12,1 & 100 \\
\hline Mujeres n & 65 & 55 & 92 & 96 & 49 & 50 & 17 & 30 & 32 & 22 & 53 & 561 \\
\hline Porcentaje & 11,6 & 9,8 & 16,4 & 17,1 & 8,7 & 8,9 & 3,0 & 5,3 & 5,7 & 3,9 & 9,4 & 100 \\
\hline Inglaterra $\mathrm{n}$ & 9 & 4 & 8 & 22 & 14 & 12 & 6 & 14 & 11 & 6 & 23 & 129 \\
\hline Porcentaje & 7,0 & 3,1 & 6,2 & 17,1 & 10,9 & 9,3 & 4,7 & 10,9 & 8,5 & 4,7 & 17,8 & 100 \\
\hline Alemania n & 4 & 8 & 9 & 8 & 5 & 2 & 3 & 4 & 5 & 4 & 9 & 61 \\
\hline Porcentaje & 6,6 & 13,1 & 14,8 & 13,1 & 8,2 & 3,3 & 4,9 & 6,6 & 8,2 & 6,6 & 14,8 & 100 \\
\hline Francia n & 1 & 2 & 3 & 7 & 3 & 7 & 1 & 5 & 2 & 3 & 9 & 43 \\
\hline Porcentaje & 2,3 & 4,7 & 7,0 & 16,3 & 7,0 & 16,3 & 2,3 & 11,6 & 4,7 & 7,0 & 20,9 & 100 \\
\hline Bélgica n & 2 & 2 & 6 & 4 & 5 & 0 & 1 & 2 & 5 & 2 & 6 & 35 \\
\hline Porcentaje & 5,7 & 5,7 & 17,1 & 11,4 & 14,3 & 0,0 & 2,9 & 5,7 & 14,3 & 5,7 & 17,1 & 100 \\
\hline España n & 1 & 1 & 7 & 2 & 3 & 4 & 2 & 3 & 3 & 3 & 9 & 38 \\
\hline Porcentaje & 2,6 & 2,6 & 18,4 & 5,3 & 7,9 & 10,5 & 5,3 & 7,9 & 7,9 & 7,9 & 23,7 & 100 \\
\hline T mínima Récord Puerto & & & \multicolumn{4}{|c|}{ 0,1으 C Bajo cero } & \multicolumn{4}{|c|}{$(04 / 02 / 2012)$} & & \\
\hline $\begin{array}{c}\text { T mínima Récord } \\
\text { Aeropuerto }\end{array}$ & & & \multicolumn{4}{|c|}{$10^{\circ} \mathrm{C}$ Bajo cero } & \multicolumn{4}{|c|}{$(12 / 02 / 1956)$} & & \\
\hline
\end{tabular}

La percepción del registro de la temperatura mínima en los turistas en general es en primer lugar de $5^{\circ} \mathrm{C}$, con la segunda percepción de $-2^{\circ} \mathrm{C}$. Según la procedencia de los países, todos coinciden en la primera percepción de temperatura mínima de $5^{\circ} \mathrm{C}$, la segunda percepción presenta variantes de respuestas. Los turistas ingleses valoran los 2 y -1 grados, los turistas alemanes valoran los -3 grados, los turistas franceses valoran los -2 y 0 grados, los turistas belgas valoran los 3 y -2 grados y los turistas españoles valoran los -3 grados. Los estudiantes captan como menor temperatura los -5 y -3 grados por orden de percepción. La percepción de los hombres es de -5 , en segundo orden de 5 y -2 grados igualitariamente. Las mujeres perciben el récord de temperatura mínima -2 grados y a continuación la temperatura de -3 grados. En los resultados de las series climáticas de las dos localizaciones de Palma, se registran las siguientes temperaturas mínimas: en el puerto se ha registrado la temperatura mínima de $-0,1^{\circ} \mathrm{C}$ y en el aeropuerto la temperatura mínima de $-10^{\circ} \mathrm{C}$. Entre las dos localizaciones se muestran registros de temperaturas, coincidentes según los principios térmicos de los efectos climáticos marítimo y continental.

Tabla 6. Percepción del día frío del año y los resultados de las series climáticas 1980-2010

\begin{tabular}{ccccccccc}
\hline DIA FRío & Los Santos & Sta Catalina & Navidad & S Antonio & S Sebastián & S Vicente & S Valentín & Total \\
\hline Estudiantes n & 16 & 13 & 52 & 84 & 90 & 38 & 30 & 323 \\
\hline Porcentaje & 5,0 & 4,0 & 16,1 & 26,0 & 27,9 & 11,8 & 9,3 & 100 \\
\hline $\begin{array}{c}\text { Media T min del día } \\
\text { Puerto }\end{array}$ & 14,4 & 10,9 & 8,7 & 8,3 & 8,2 & 8,4 & 8,3 & \\
\hline $\begin{array}{c}\text { Récord T min media } \\
\text { del día Puerto }\end{array}$ & & \multicolumn{7}{c}{5 de enero - (7,6-0 $)$} \\
\hline
\end{tabular}


La percepción de los estudiantes de conocer el día con las temperaturas mínimas medias más bajas del año tiene su impresión principalmente en el día de San Sebastián, la segunda percepción es el día de San Antonio, seguida del día de Navidad. En los resultados de las series climáticas de los días expresados el de menor temperatura mínima media es el día de San Sebastián, seguido en escasa diferencia con el día de San Antonio y San Valentín. De la serie analizada el día con la menor temperatura mínima media es el día 5 de enero, con $7,6^{\circ} \mathrm{C}$, siendo el día más frío del año de la serie.

Tabla 7. Percepción del día de calor del año y los resultados de las series climáticas 1980-2010

\begin{tabular}{ccccccc} 
DíA CALOR & S Juan & Sta Margarita & Sta Ana & S Lorenzo & S Bernardo & Total \\
\hline Estudiantes n & 72 & 64 & 54 & 121 & 14 & 325 \\
\hline Porcentaje & 22,2 & 19,7 & 16,6 & 37,2 & 4,3 & 100 \\
\hline $\begin{array}{c}\text { T máxima media del día } \\
\text { Puerto }\end{array}$ & 28,1 & 29,6 & 29,9 & 29,3 & 30,3 & \\
\hline $\begin{array}{c}\text { Récord Tm máxima del } \\
\text { día Puerto }\end{array}$ & & 12 de agosto - (30,3ำ & \\
\hline
\end{tabular}

La percepción de los estudiantes de conocer el día con las temperaturas máximas medias más altas del año tienen su impresión principalmente en el día de San Lorenzo, la segunda percepción es el día de San Juan, seguido del día se Santa Margarita. En los resultados de las series climáticas de los días expresados el de mayor temperatura máxima media es el día San Bernardo, el segundo el día de Santa Ana y el tercero el día de Santa Margarita. De la serie analizada el día con la mayor temperatura media es el día 12 de agosto, con 30,3ํㅡ, siendo el día de más calor del año de la serie.

Tabla 8. Percepción de la hora con la temperatura máxima diaria y los resultados de las series climáticas 1980-2010

\begin{tabular}{|c|c|c|c|c|c|c|c|c|c|c|c|c|c|}
\hline HORA MAX TEMPERATURA & 9 & 10 & 11 & 12 & 13 & 14 & 15 & 16 & 17 & 18 & 19 & 20 & Total \\
\hline Turistas $\mathrm{n}$ & 1 & 1 & 1 & 34 & 112 & 118 & 71 & 26 & 14 & 10 & 3 & 17 & 408 \\
\hline Porcentaje & 0,2 & 0,2 & 0,2 & 8,3 & 27,5 & 28,9 & 17,4 & 6,4 & 3,4 & 2,5 & 0,7 & 4 & 100 \\
\hline Estudiantes n & 1 & 0 & 0 & 57 & 48 & 97 & 104 & 21 & 5 & 2 & 0 & 2 & 337 \\
\hline Porcentaje & 0,3 & 0,0 & 0,0 & 16,9 & 14,2 & 28,8 & 30,9 & 6,2 & 1,5 & 0,6 & 0,0 & 0,6 & 100 \\
\hline Hombres n & 0 & 1 & 1 & 43 & 74 & 108 & 93 & 28 & 14 & 8 & 2 & 8 & 380 \\
\hline Porcentaje & 0,0 & 0,3 & 0,3 & 11,3 & 19,5 & 28,4 & 24,5 & 7,4 & 3,7 & 2,1 & 0,5 & 2,1 & 100 \\
\hline Mujeres n & 1 & 0 & 0 & 78 & 106 & 162 & 151 & 22 & 6 & 6 & 7 & 12 & 551 \\
\hline Porcentaje & 0,2 & 0,0 & 0,0 & 14,2 & 19,2 & 29,4 & 27,4 & 4,0 & 1,1 & 1,1 & 1,3 & 2,2 & 100 \\
\hline Inglaterra n & 0 & 0 & 0 & 10 & 39 & 35 & 21 & 11 & 2 & 8 & 0 & 7 & 133 \\
\hline Porcentaje & 0,0 & 0,0 & 0,0 & 7,5 & 29,3 & 26,3 & 15,8 & 8,3 & 1,5 & 6,0 & 0,0 & 5,3 & 100 \\
\hline Alemania $\mathrm{n}$ & 0 & 0 & 0 & 6 & 18 & 20 & 10 & 3 & 2 & 0 & 1 & 5 & 65 \\
\hline Porcentaje & 0,0 & 0,0 & 0,0 & 9,2 & 27,7 & 30,8 & 15,4 & 4,6 & 3,1 & 0,0 & 1,5 & 7,7 & 100 \\
\hline Francia $\mathrm{n}$ & 1 & 0 & 0 & 5 & 7 & 15 & 6 & 2 & 1 & 0 & 0 & 1 & 38 \\
\hline Porcentaje & 2,6 & 0,0 & 0,0 & 13,2 & 18,4 & 39,5 & 15,8 & 5,3 & 2,6 & 0,0 & 0,0 & 2,6 & 100 \\
\hline Bélgica n & 0 & 0 & 1 & 1 & 9 & 9 & 7 & 3 & 2 & 1 & 0 & 1 & 34 \\
\hline Porcentaje & 0,0 & 0,0 & 2,9 & 2,9 & 26,5 & 26,5 & 20,6 & 8,8 & 5,9 & 2,9 & 0,0 & 2,9 & 100 \\
\hline España n & 0 & 0 & 0 & 2 & 8 & 10 & 10 & 3 & 2 & 0 & 1 & 2 & 38 \\
\hline Porcentaje & 0,0 & 0,0 & 0,0 & 5,3 & 21,1 & 26,3 & 26,3 & 7,9 & 5,3 & 0,0 & 2,6 & 5,3 & 100 \\
\hline Media de la hora Max T Puerto & \multicolumn{13}{|c|}{$12: 26$} \\
\hline $\begin{array}{l}\text { Media de la hora max T } \\
\text { Aeropuerto }\end{array}$ & \multicolumn{13}{|c|}{$12: 42$} \\
\hline
\end{tabular}


La percepción general de los turistas en base a la hora del día en que se registran las mayores temperaturas, son las 14 horas como primera impresión y las 13 horas como segunda impresión. Los turistas de Reino Unido valoran en primer orden las 13 horas y después las 14 horas, como las de mayor sensación de calor. Los turistas de Alemania y Francia perciben primero las 14 horas y en segunda opción las 13 horas. Los turistas belgas coinciden en ambas horas del día, las 13 y $14 \mathrm{~h}$, como las de altas temperaturas. Los turistas de España, valoran las 15 y 14 horas, como las horas de temperaturas altas diarias. Los estudiantes perciben las 15 y 14 horas, respectivamente. Los hombres y las mujeres perciben las 14 y 15 horas. En los resultados de las series climáticas reflejan la media de las horas en que se han producido las temperaturas máximas del día, en el puerto a las 12:26 h y en el aeropuerto a las 12:42 h. Las horas del mediodía son las más calurosas. En el aeropuerto la media de la hora de máxima temperatura se presenta 16 minutos más tarde que en el puerto.

Tabla 9. Percepción de la hora con la temperatura mínima diaria y los resultados de las series climáticas 1980-2010

\begin{tabular}{|c|c|c|c|c|c|c|c|c|c|c|c|c|c|}
\hline HORA MIN TEMPERATURA & 21 & 22 & 23 & $\mathbf{0}$ & 1 & 2 & 3 & 4 & 5 & 6 & 7 & 8 & Total \\
\hline Turistas n & 14 & 10 & 18 & 31 & 28 & 47 & 76 & 53 & 42 & 28 & 14 & 18 & 379 \\
\hline Porcentaje & 3,7 & 2,6 & 4,7 & 8,2 & 7,4 & 12,4 & 20,1 & 14,0 & 11,1 & 7,4 & 3,7 & 4,7 & 100 \\
\hline Estudiantes n & 2 & 4 & 15 & 38 & 18 & 35 & 48 & 65 & 50 & 44 & 10 & 12 & 341 \\
\hline Porcentaje & 0,6 & 1,2 & 4,4 & 11,1 & 5,3 & 10,3 & 14,1 & 19,1 & 14,7 & 12,9 & 2,9 & 3,5 & 100 \\
\hline Hombres n & 9 & 6 & 8 & 27 & 23 & 42 & 62 & 81 & 47 & 45 & 15 & 10 & 375 \\
\hline Porcentaje & 2,4 & 1,6 & 2,1 & 7,2 & 6,1 & 11,2 & 16,5 & 21,6 & 12,5 & 12,0 & 4,0 & 2,7 & 100 \\
\hline Mujeres n & 11 & 12 & 28 & 55 & 36 & 64 & 87 & 104 & 67 & 49 & 18 & 24 & 555 \\
\hline Porcentaje & 2,0 & 2,2 & 5,05 & 9,91 & 6,5 & 11,5 & 15,7 & 18,7 & 12,1 & 8,83 & 3,2 & 4,3 & 100 \\
\hline Inglaterra $\mathrm{n}$ & 8 & 7 & 7 & 15 & 10 & 16 & 23 & 19 & 7 & 6 & 3 & 11 & 132 \\
\hline Porcentaje & 6,1 & 5,3 & 5,3 & 11,4 & 7,6 & 12,1 & 17,4 & 14,4 & 5,3 & 4,5 & 2,3 & 8,3 & 100 \\
\hline Alemania n & 1 & 2 & 0 & 7 & 3 & 14 & 13 & 10 & 8 & 3 & 2 & 1 & 64 \\
\hline Porcentaje & 1,6 & 3,1 & 0,0 & 10,9 & 4,7 & 21,9 & 20,3 & 15,6 & 12,5 & 4,7 & 3,1 & 1,6 & 100 \\
\hline Francia n & 0 & 0 & 23 & 1 & 5 & 3 & 10 & 11 & 7 & 1 & 0 & 1 & 62 \\
\hline Porcentaje & 0,0 & 0,0 & 37,1 & 1,6 & 8,1 & 4,8 & 16,1 & 17,7 & 11,3 & 1,6 & 0,0 & 1,6 & 100 \\
\hline Bélgica n & 2 & 0 & 0 & 0 & 3 & 5 & 10 & 9 & 1 & 1 & 1 & 2 & 34 \\
\hline Porcentaje & 5,9 & 0,0 & 0,0 & 0,0 & 8,8 & 14,7 & 29,4 & 26,5 & 2,9 & 2,9 & 2,9 & 5,9 & 100 \\
\hline España n & 2 & 0 & 4 & 4 & 2 & 1 & 4 & 7 & 8 & 3 & 3 & 0 & 38 \\
\hline Porcentaje & 5,3 & 0,0 & 10,5 & 10,5 & 5,3 & 2,6 & 10,5 & 18,4 & 21,1 & 7,9 & 7,9 & 0,0 & 100 \\
\hline Media de la hora min T Puerto & \multicolumn{13}{|c|}{$8: 18$} \\
\hline Media de la hora min T Aeropuerto & \multicolumn{13}{|c|}{$8: 13$} \\
\hline
\end{tabular}

La percepción general de los turistas en base a la hora del día en que se registran las menores temperaturas, son a las 3 horas de madrugada como primera impresión y a las 4 horas de la madrugada como segunda impresió n. Los turistas de Reino Unido y de Bélgica valoran en primer orden las 3 horas y después las 4 horas, como las de mayor sensación de frío. Los turistas de Alemania perciben como primera opción las 2 horas y en segunda opción las 3 horas. Los turistas de Francia tienen la idea de ser a las 23 horas y a continuación las segundas opiniones a las 4 horas. Los turistas de España, valoran las 5 horas y las 4 horas, como el periodo del día con bajas temperaturas. Los estudiantes perciben las 4 y 5 de la madrugada. Los dos sexos encuestados coinciden en que las temperaturas más bajas del día son a las 4 y 3 de madrugada, respectivamente. Los resultados de las series climáticas reflejan la media de las horas en que se han producido las temperaturas mínimas del día, en el puerto a las 8:18 horas 
y en el aeropuerto a las 8:13 horas. Las horas de temperaturas mínimas son a primera hora de la mañana, después del amanecer con escasa diferencia entre ambas localizaciones.

\section{CONCLUSIÓN I DISCUSIÓN}

Los datos analizados de la serie climatológica del puerto de Porto Pi y del Aeropuerto en la ciudad de Palma, muestran el siguiente análisis. El mes más frío del año en el puerto es enero con $8,3^{\circ} \mathrm{C}$ y en el aeropuerto es el mes de enero con 3,8ㅜ․ El mes más calurosos del año en el puerto es el mes de agosto con $29,8^{\circ} \mathrm{C}$ y en el aeropuerto julio con $31,3^{\circ} \mathrm{C}$. La mayor oscilación térmica es de 7,6ㄷ (Puerto), en junio y marzo, y de 12,9 (Aeropuerto), en el mes de julio. En referencia a la temperatura máxima absoluta registrada es de $38.0^{\circ} \mathrm{C}$ el día 21 julio de 2003 (Puerto) y de 41,4ํㅡ el 25 de junio de 2011 (Aeropuerto). La temperatura media de las máximas más altas es de $33.7^{\circ} \mathrm{C}$, el mes de agosto de 2003. La temperatura media más alta es de $29.4^{\circ} \mathrm{C}$, el mes de agosto 2003 . La temperatura mínima absoluta es de $-0.1^{\circ} \mathrm{C}$ bajo cero, el día de 04 febrero de 2012 (Puerto) y de $-10^{\circ} \mathrm{C}$ bajo cero el día 12 de febrero de 1956 (Aeropuerto). El récord de la temperatura mínima media anual en el puerto es de 7,6ํㅡ, acontecido en el día 5 de enero, se considera analíticamente el día más frío del año. El récord de la temperatura media máxima anual en el puerto es de $30,3^{\circ} \mathrm{C}$, produciéndose el día 12 de agosto, considerado estadísticamente el día más caluroso del año. Respecto a las horas con epicentros de cambios térmicos de bajas y altas temperaturas. Se ha analizado y destaca que a las ocho de la mañana se registran las temperaturas más bajas de la jornada y que a las doce del mediodía se registran las temperaturas más altas de las jornadas.

En la consideración de la percepción, las temperaturas frías están percibidas en mayor grado por la comunidad española, la comunidad de estudiantes, la comunidad francesa y la comunidad inglesa. Es importante destacar que la vinculación al territorio, tanto por estudios, residencia o vinculación geográfica determina de manera destacada la realidad climatológica de la isla de Mallorca. Si en el caso, se considera la percepción de las temperaturas altas, se analiza que se aprecia mayor grado de percepción en la comunidad de estudiantes, en la comunidad alemana y en la comunidad francesa. Las temperaturas altas son propias de la estación veraniega, es por dicha razón que el turista puede percibir dichas temperaturas de modo más fiables. En cambio en el transcurso de las temperaturas bajas, en cuyo curso anual, el turismo no visita las islas. En la percepción de las temperaturas es importante destacar que se incluyen factores vinculantes como la residencia en la isla, la experiencia de visitas anteriores, la percepción en las fechas del viaje y la influencia de los parte meteorológicos en los medios de comunicación.

\section{BIBLIOGRAFÍA}

Alberola, A. (1996): “La percepción de la catástrofe: Sequía e inundaciones en tierras valencianas durante la primera mitad del siglo XVIII". Revista De Historia Moderna, n.15 (1996); pp.257-269-

Alomar, G. et al (2010): “La percepción geográfica del régimen de brisas en Mallorca. De la experiencia directa a los datos instrumentales". Departamento de la Universidad de las Islas Baleares.

Andrade, H. et al. (2011): "Perception of temperature and wind by users of public outdoor spaces: Relationships with weather parameters and personal characteristics". International Journal ofBiometeorology, 55(5), pp 665680.-

Balling Jr, - Cerveny, R. (2003). “Compilation and discussion of trends in severe storms in the United States: Popular perception v. climate reality”. Natural Hazards, 29(2), pp 103-112.

Bellés, J. (1990). “Avenidas y riesgos de inundación en los sistemas fluviales mediterráneos de la Península Ibérica”. Boletín de la Asociación de Geógrafos Españoles, (10), pp 45-86.

Capstick, S, - Pidgeon, N. (2013): "Public perception of cold weather events as evidence for and against climate change". Climatic Change. Pp 1-14. 
Calleja, J. M. T. (2020). "Percepción de la Temperatura Climatológica en el Turismo y en Residentes de la Isla de Mallorca". Advances in Social Sciences Research Journal, 7(1) 236-246.

Changnon Jr. et al (1971): "METROMEX: An investigation of inadvertent weather modification”. Bulletin of the American Meteorological Society, 52(10), 958-968.-Ciencia, Medicina, Comunicación y Cultura, (37), pp 65-70.

González, I. L. (2009): “La percepción del clima de Ávila”. Aula, 7

López, F. (1995): “Nota sobre la percepción del clima urbano. el ejemplo de la ciudad de Zaragoza”. Geographicalia, (32), pp 123-137.

López, F. Saz, M.A. "La problemática de la percepción y comunicación social de los fenómenos climáticos”.

March, H, et al (2014). "Rising Temperatures and Dwindling Water Supplies? Perception of Climate Change Among Residents of the Spanish Mediterranean Tourist Coastal Areas”. Environmental management, 53(1), pp 181-193.

Martín Vide, J. (1990). “La percepción del clima en las ciudades”.

Moreno, MC. (1987): “Frecuencias de la precipitación según los días de la semana en Barcelona”. Revista De Geografia, 21(1), pp 5-10.

Sánchez, M. L. (2006): “La divulgación científica de la meteorología: Emisores implicados”.

Semenza, J. et al. (2008). "Public perception of climate change: voluntary mitigation and barriers to behavior change". American journal of preventive medicine, 35(5), pp 479-487. 